\title{
Excited-state Dynamics of Radical lons in Liquids
}

\author{
Jakob Griljab, Joseph S. Beckwith ${ }^{\mathrm{ac}}$, and Eric Vauthey ${ }^{\star a}$
}

\begin{abstract}
Thomas Bally has acquired international recognition for his work on the photochemistry of reactive intermediates, which include radical ions. Here, we present a brief overview of our investigations of the excited-state dynamics of radical ions in liquids at room temperature, which are still poorly documented. A better understanding of these dynamics is most relevant, as open-shell ions in the excited state are being increasingly used in redox photochemistry and have been proposed to play a key role in highly exergonic photoinduced electron transfer reactions.
\end{abstract}

Keywords: Electron transfer · Photochemistry · Photophysics · Ultrafast spectroscopy

Jakob Grilj obtained his diploma from Graz University of Technology (1998) and his PhD from University of Geneva (2011). After postdoctoral work at Stanford University's Linear Accelerator Center, at EPF Lausanne, and the Paul Scherrer Institute, he quit academic research.

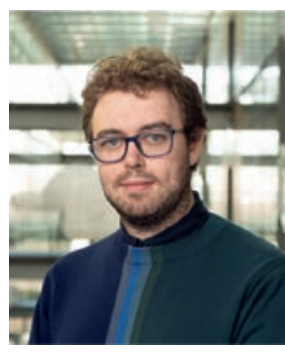

Joseph Beckwith obtained his undergraduate degree in chemistry from Durham University in 2013. After an MPhil with David Klenerman at the University of Cambridge (2014) he obtained his $\mathrm{PhD}$ in the group of Eric Vauthey in 2019. Since 2020 he has been an SNF Early Postdoctoral Fellow in the group of Haw Yang at Princeton, developing and using single-particle spectroscopies to understand complex systems.

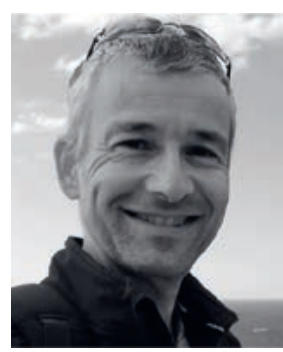

Eric Vauthey obtained his chemistry diploma (1986) and PhD (1989) from the University of Fribourg. After postdoctoral research at Imperial College London and ETH Zurich, he started independent research in Fribourg in 1992. He has been a Professor at the University of Geneva since 2001. His main research interest is the dynamics of molecular photoinduced processes in the condensed phase and at interfaces

\section{Introduction}

Open-shell ions are ubiquitous reactive intermediates in chemistry. It has been quickly realised that their reactivity can be significantly boosted upon optical excitation. [1] For example, Moutet and Reverdy reported on the oxidation of benzyl alcohol to benzaldehyde by the radical cation of N,N,N',N'-tetraphenyl$p$-phenylenediamine in the excited state. ${ }^{[2]}$ Since then, the spectacular reactivity of photo-excited radical ions has been attracting increasing interest. ${ }^{[3]}$

Radical ions in the electronic excited state have also been invoked to account for the absence of the inverted region in bi- molecular photoinduced electron transfer (ET) reactions between two neutral reactants. ${ }^{[4]}$ ET quenching is well known to be diffusion-controlled ET at driving forces up to $2.5 \mathrm{eV}$ where Marcus theory predicts a slowing down of the reaction. This apparent contradiction to experiment ${ }^{[4]}$ could be explained by the formation of the radical ions in an electronic excited-state, which are generally low for these species. ${ }^{[5]}$ Although this hypothesis was proposed more than 50 years ago, ${ }^{[4 a]}$ unambiguous experimental observation of the formation of excited ions upon highly exergonic ET is still lacking.

Despite the clear relevance of excited radical ions for different areas of photochemistry, their dynamics are still poorly understood. Our interest in this field goes back several decades while at the Institute of Physical Chemistry in Fribourg, where Thomas Bally was investigating the photochemistry of reactive intermediates, including radical ions, in low-temperature solid matrices. ${ }^{[6]}$ Our first studies were mostly performed in solids, either in room-temperature boric acid glass or in low-temperature matrices. ${ }^{[7]}$ Using transient grating-based methods, we could show that the excited-state lifetime of a few radical cations, perylene and thioanthrene as well as anthraquinone, in low-temperature glasses was ranging from 30 to 100 ps. In room-temperature liquids, however, the lifetimes were shorter than the $\sim 25$ ps time resolution of our set-up at that time.

In the following, we give an overview of our more recent studies of the excited-state dynamics of radical ions in liquids using femtosecond spectroscopy.

\section{Experimental Approaches}

The main obstacle to the study of radical ions stems precisely from their transient nature and high reactivity. Here, we briefly discuss our approaches to generate radical ions at sufficient concentration and stability to perform femtosecond transient electronic absorption and, in some cases, fluorescence up-conversion measurements.

A limited number of radical cations, including the well-known Wurster's Blue, the radical cation of $\mathrm{N}, \mathrm{N}, \mathrm{N}^{\prime}, \mathrm{N}^{\prime}$-tetramethyl- $p$ phenylenediamine (TMPD $\left.{ }^{+}\right)$, can be prepared as stable salts that can be kept as solids in normal conditions for months or longer. [8] The study of these 'ideal' radical ions does not require significantly more caution than that of conventional closed-shell dyes. 
- A few aromatic hydrocarbons, such as those of perylene and tetracene, can be transformed into their corresponding radical cation in concentrated sulfuric acid, that acts as a strong oxidising medium. ${ }^{[9]}$ Although this is a simple and convenient way to produce relatively stable solutions of radical cations, it should be kept in mind that sulfuric acid is not a well-defined medium and comprises a dynamic equilibrium involving several species. Therefore, occurrence of reactions between the excited cations and some of these species cannot be totally ruled out.

- Whereas electrochemistry is the most evident way to produce radical ions, its combination with ultrafast spectroscopy is not straightforward. Spectroscopic measurements require that only the ions absorb at the excitation wavelength and that the sample at the irradiation spot is rapidly refreshed. Therefore, standard optically-transparent thin layer electrochemical (OTTLE) cells, ${ }^{[10]}$ such as those used routinely for stationary spectro-electrochemistry are not suited. Instead, we used an electrochemical flow cell similar to that reported in ref. [11] and located just before the optical cell.[12] This approach was successful with a limited number of compounds. In many cases, continuous and steady production of ions over the whole duration of a pump-probe experiment proved to be problematic.

- Finally, the above complications encountered with the electrochemical generation can be avoided by producing the ions via a bimolecular photoinduced ET reaction. For this, we performed so-called pump-pump-probe measurements (Fig. 1): first, a pump pulse, the actinic pulse, triggers the photoinduced ET process to generate radical ions, and after a time delay long enough to ensure a sufficient concentration of ions, a 'conventional' pump-probe experiment is performed on one of the two ensuing ions. ${ }^{[13]}$ The advantage of this approach is that it allows better tuning of the solvent, contrary to sulfuric acid or to the electrochemical preparation. However, performing transient absorption on a transient species remains challenging and requires a setup with high sensitivity and wavelength tunability.

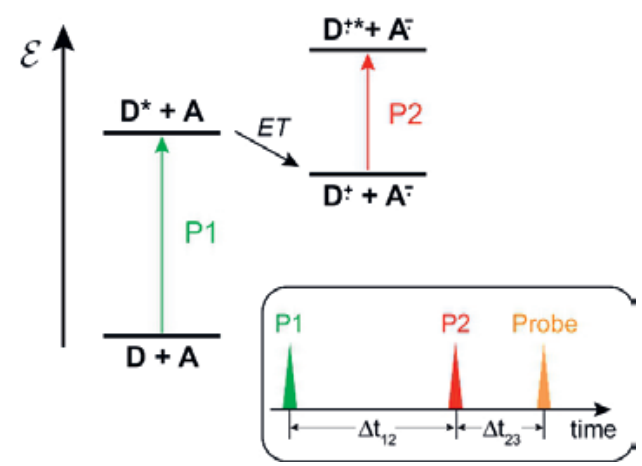

Fig. 1. Principle of the pump-pump-probe method for investigating the excited-state dynamics of radical ions. The pump pulse P1 excites locally one of the two reactants (here the donor) to trigger the electron transfer (ET). After a time interval $\Delta t_{12}$, the pump pulse P2 excites one of the two resulting radical ions (here the cation) and an ensuing broadband probe pulse measures the absorption changes over the whole visible region at different time delays $\Delta t_{23}$.

\section{The Radical Cations of Aromatic Amines}

Fig. 2 shows the time evolution of the $\mathrm{D}_{1} \rightarrow \mathrm{D}_{0}$ emission spectrum measured with a solution of Wurster's Blue, TMPD ${ }^{+}$(Fig. 3 ), using fluorescence up-conversion spectroscopy (FLUPS) ${ }^{[14]}$ in acetonitrile at room temperature. The decay of the emission band is extremely rapid and corresponds to a fluorescence lifetime of $240 \mathrm{fs}$. This ultrashort lifetime is consistent with a fluorescence quantum yield below the detection limit of stationary fluorescence spectroscopy. The same excited-state lifetime is obtained from transient electronic absorption measurements, ${ }^{[15]}$ that point to a rapid $D_{1} \rightarrow D_{0}$ internal conversion followed by the vibrational cooling of the hot ground state. These excited-state dynamics, discussed in detail in ref. [15], were found to be essentially the same in a large variety of solvents including chloroform, water and heavy water, as well as a viscous room-temperature ionic liquid. However, these dynamics were found to strongly slow down upon decreasing temperature. As a consequence, the fluorescence lifetime of TMPD ${ }^{+}$below $140 \mathrm{~K}$ is sufficiently slow, $660 \mathrm{ps}$ at $77 \mathrm{~K}$, for the $\mathrm{D}_{1} \rightarrow \mathrm{D}_{0}$ emission to be visible by stationary fluorescence.

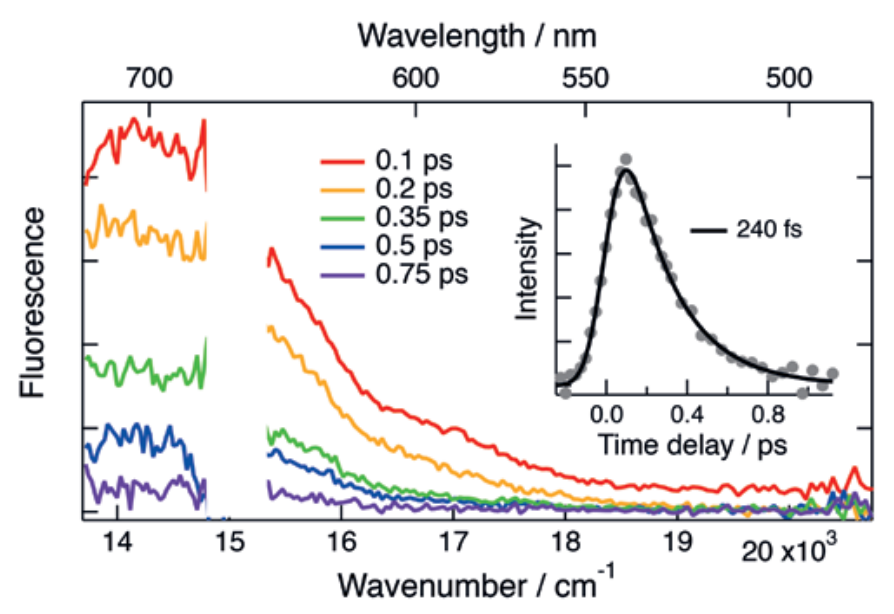

Fig. 2: Time-resolved fluorescence spectra measured at various time delays after excitation of a solution of Wurster's Blue (TMPD.+) in acetonitrile. Inset: time profile of the fluorescence intensity at $700 \mathrm{~nm}$ $\left(14300 \mathrm{~cm}^{-1}\right)$ and best fit of an exponential function with the instrument response function.

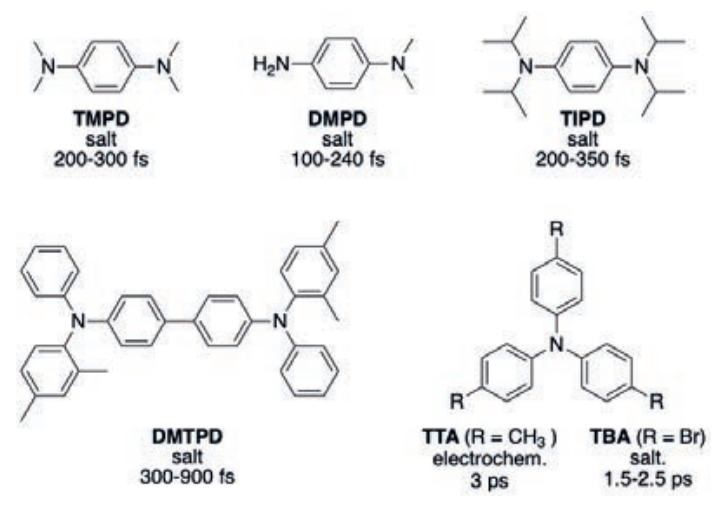

Fig. 3. Structure of the aromatic amines, whose radical cations (formed as a salt or electrochemically) were investigated, together with the excited-state lifetimes in liquids at room temperature determined by ultrafast spectroscopy.

The ultrafast $\mathrm{D}_{1} \rightarrow \mathrm{D}_{0}$ internal conversion at room temperature and the strong temperature dependence were explained by the presence of a $\mathrm{D}_{1} / \mathrm{D}_{0}$ conical intersection $(\mathrm{CI})$ separated from the Franck-Condon region of the $\mathrm{D}_{1}$ state by a small barrier. This hypothesis was supported by quantum-chemical calculations performed by the Olivucci group suggesting that access to the CI involves the rotation of the dimethyl-amino groups relative to the phenyl ring. ${ }^{[15]}$ To test whether access to this CI could be influenced by varying the size of the amino substituents, we performed similar measurements with the radical cation of N,N-dimethylp-phenylenediamine (DMPD $\left.{ }^{+}\right)$and N,N,N',N'-tetraisopropyl-pphenylenediamine $\left(\text { TIPD }^{{ }^{+}}\right)^{[8 c]}$ Not very surprisingly, replacement of two methyl groups by $\mathrm{H}$ atoms in DMPD ${ }^{+}$did not result in very significant changes in the excited-state dynamics. More surprising was the observation that the replacement of all four me- 
thyl groups by bulky isopropyl groups had no effect either on the excited-state lifetime of TiPD ${ }^{+}$. Furthermore, whereas stationary fluorescence could be observed with DMPD ${ }^{+}$below $120 \mathrm{~K}$, no emission could be detected with $\mathbf{T i P D}{ }^{+}$, even at a temperature as low as $12 \mathrm{~K}$. It was thus concluded that, although the twist of the amino groups is significantly hindered in TiPD ${ }^{+}$, other $\mathrm{D}_{1} / \mathrm{D}_{0} \mathrm{CIs}$ should probably be accessible via different coordinates, such as the pyramidalisation of the nitrogen centres or the deformation of the phenyl ring.

Ultrafast excited-state dynamics seem to be a general feature of the radical cations of aromatic amines. Fig. 4 shows transient absorption spectra recorded with the radical cation of N,N'-diphenyl-N,N'-bis(2,4-dimethylphenyl)-1,1'-biphenyl-4,4'diamine (DMTPD $\left.{ }^{+}\right)$in acetonitrile after $500 \mathrm{~nm}$ excitation, together with evolution-associated difference absorption spectra (EADS) obtained from a global analysis of the data assuming a succession of exponential steps. The absorption below $400 \mathrm{~nm}$ and above $600 \mathrm{~nm}$ can be assigned to $\mathrm{D} \leftarrow \mathrm{D}$ transitions, whereas the band on the low energy side of the bleach can be attributed to the vibrationally hot ground state. This data suggest an $D_{1} \rightarrow D_{0}$ internal conversion in about 300 fs followed by vibrational cooling on the 1.2 and 15 ps timescale. ${ }^{[12]}$

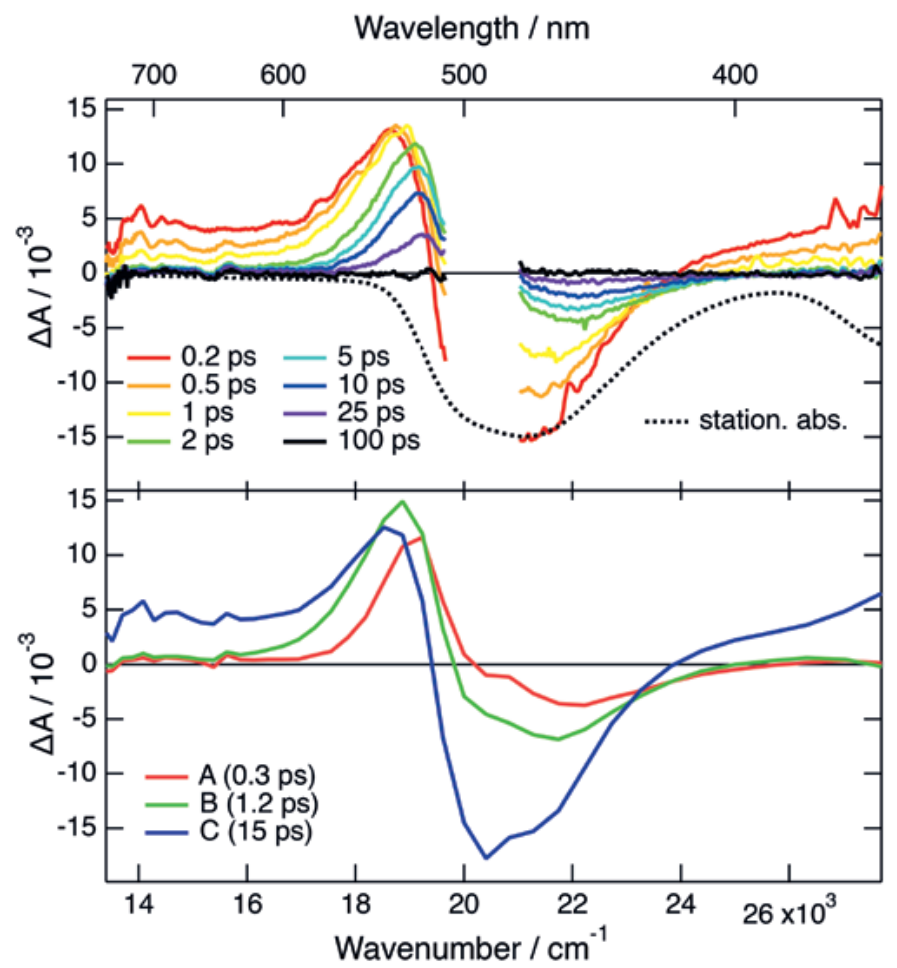

Fig. 4. Transient absorption spectra recorded at various time delays after $500 \mathrm{~nm}$ excitation of DMTPD.+ in acetonitrile and negative stationary absorption spectrum (Top). Evolution-associated difference absorption spectra (EADS) and corresponding time constants obtained from a global analysis of the transient absorption data assuming a series of three successive exponential steps, $A \rightarrow B \rightarrow C \rightarrow$ (Bottom). $A$ can be assigned to DMTPD.+ in the $D_{1}$ state, and $B$ and $C$ to the vibrationally hot $D_{0}$ state. Some contribution of the D1 state to EADS $B$ arising from nonexponential dynamics cannot be excluded.

Qualitatively similar behaviour was observed with the radical cations of two triarylamines in acetonitrile, TTA ${ }^{+}$and $\mathbf{T B A} \mathbf{}^{+}$, the latter being also known as Magic Blue, i.e. sub-picosecond $\mathrm{D}_{1} \rightarrow \mathrm{D}_{0}$ internal conversion followed by vibrational relaxation on timescales ranging from $1-2$ ps to about 15 ps. ${ }^{[12]}$

It should be noted that all three DMTPD, TTA and TBA are flexible molecules already in the neutral closed-shell form with low barrier for torsion around the $\mathrm{C}-\mathrm{N}$ bonds between the central

$\mathrm{N}$ atom and the phenyl substituents. This flexibility can be expected to increase further upon oxidation, i.e. after removal of an electron from a bonding orbital, and upon electronic excitation. It is therefore not a coincidence that the excited-state dynamics of these cations is very similar to that reported for triarylmethane cationic dyes, such as Malachite Green, whose $S_{1}$ state decays in less than $1 \mathrm{ps}$ in acetonitrile through a rapid internal conversion via a $\mathrm{S}_{1} / \mathrm{S}_{0} \mathrm{CI}$ accessed through torsional motion of the phenyl substituents. ${ }^{[16]}$ Yet, whereas the excited-state decay of these closed-shell cationic dyes slow down considerably with increasing solvent viscosity, no significant viscosity dependence was found with the triarylamine cations, suggesting the presence of $\mathrm{D}_{1} / \mathrm{D}_{0} \mathrm{CI}(\mathrm{s})$ that can be reached without large-amplitude motion.

\section{The Radical Ions of Polycyclic Aromatic Hydrocarbons}

The stationary spectroscopy of polycyclic aromatic hydrocarbon (PAHs) ions has been intensively studied already in the 1960 s and 1970s, both in the gas and condensed phases.[5a,17] It was rapidly realised that emission from these open-shell species was scarce. This was usually explained by their generally low $\mathrm{D}_{1}-\mathrm{D}_{0}$ gap, favouring fast internal conversion and slow radiative decay in the near IR region. Interestingly, PAH cations, such as perylene radical cation (Pe ${ }^{+}$, Fig. 5), were proposed to be responsible for the emissive diffuse interstellar bands. ${ }^{[18]}$ The first investigations of the excited-state dynamics of $\mathrm{PAH}$ cations were performed with perylene, pyrene, anthracene and naphthalene radical cations in boric acid glasses at room temperature. ${ }^{[7 a, 19]}$ For all these cations, except for $\mathbf{P e}^{{ }^{+}}$, ultrafast sub-ps internal conversion was reported.[19] On the other hand, the $\mathrm{D}_{1}$ state lifetime of $\mathbf{P e}{ }^{{ }^{+}}$was significantly longer, of the order of 20 to 35 ps. ${ }^{[7 a, 19]}$

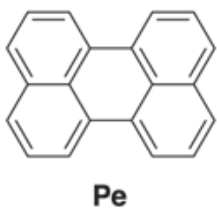

$\mathrm{Pe}$

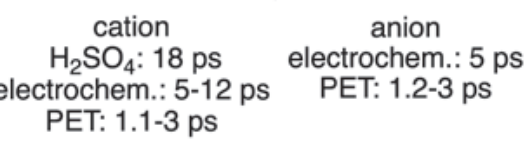

Fig. 5. Structure of the polycyclic aromatic hydrocarbons, whose radical cations or anions (formed in sulphuric acid, electrochemically or through a photoinduced electron transfer, PET) were investigated, together with the excited-state lifetimes in liquids at room temperature determined by ultrafast spectroscopy.

Fig. 6 shows transient absorption spectra recorded at various

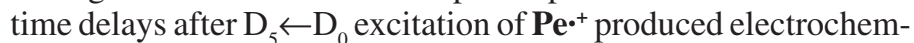
ically in acetonitrile. ${ }^{[12]}$ Qualitatively similar spectra and dynamics were observed upon $\mathrm{D}_{1} \leftarrow \mathrm{D}_{0}$ excitation at $800 \mathrm{~nm}$. Therefore, the positive bands below $500 \mathrm{~nm}$ can be assigned to the $\mathrm{D}_{1}$ state, which decays in 5 ps to the vibrationally hot $\mathrm{D}_{0}$ state, which in turns thermalises in about 25 ps. Perdeuteration or substitution in the 3 position by a methyl group has no marked effect on the dynamics. However, the $\mathrm{D}_{1}$ state lifetime increases to $12 \mathrm{ps}$ and 18 ps upon going to $\mathrm{CH}_{2} \mathrm{Cl}_{2}$ and sulfuric acid, respectively. ${ }^{[12]}$ This effect points to a possible influence of the solvent viscosity on the excited-state dynamics, in agreement with the 20-35 ps lifetime reported in boric acid. ${ }^{[7 \mathrm{a}, 19]}$ Trifunac and coworkers explained the longer excited-state lifetime of $\mathbf{P e}^{+}$compared to the other investigated PAH cations by the absence of any easily accessible $\mathrm{D}_{1} /$ $\mathrm{D}_{0}$ CI. ${ }^{[19]}$ Quantum-chemical calculations by Robb and coworkers on the radical cations of naphthalene and pyrene indeed point to the existence of $\mathrm{D}_{1} / \mathrm{D}_{0} \mathrm{CI}$ that can be easily reached via in-plane 
distortion. ${ }^{[20]}$ The same group explained the longer excited-state lifetime of $\mathbf{P e}^{+}$in boric acid by the absence of such a CI. ${ }^{[21]}$ The shorter lifetime found by us in liquid media and its apparent dependence on viscosity points to the possible role of another $\mathrm{D}_{1} / \mathrm{D}_{0} \mathrm{CI}$ that could involve out-of-plane deformation. However, $\mathbf{P e}^{+}$was restricted to a planar geometry in the calculations of Robb and coworkers preventing the detection of CIs associated with out-of-plane distortion.

$\mathrm{A} \mathrm{D}_{1}$ lifetime of $23 \mathrm{ps}$ was measured with the radical cation of tetracene, $\mathbf{T e}^{+}$, in sulfuric acid. ${ }^{[12]}$ This value is close to that found with $\mathbf{P e}^{+}$in the same medium. Unfortunately, electrochemical production of $\mathbf{T e}^{+}$was not successful, preventing the possibility to explore the solvent dependence of the excited-state dynamics.

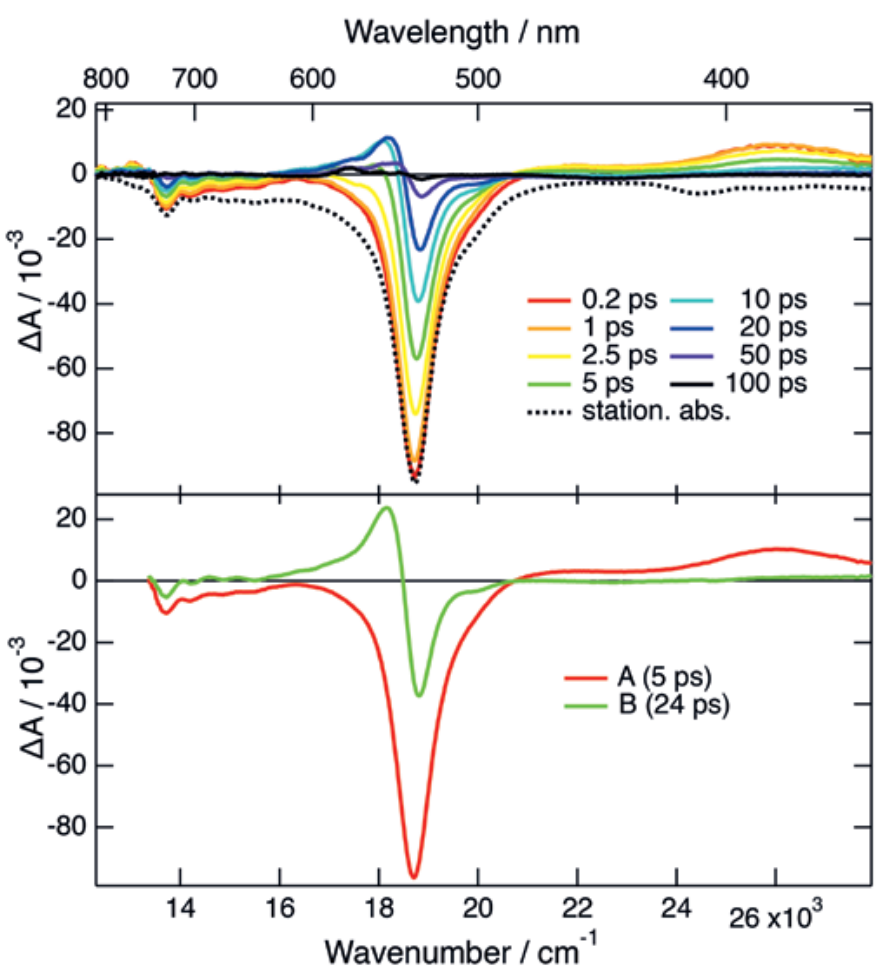

Fig. 6. Transient absorption spectra recorded at various time delays after $500 \mathrm{~nm}$ excitation of $\mathbf{P e} \cdot{ }^{+}$produced electrochemically in acetonitrile/ $\mathrm{Bu}_{4} \mathrm{NPF}_{6}$ and negative stationary absorption spectrum (Top). Evolutionassociated difference absorption spectra (EADS) and corresponding time constants obtained from a global analysis of the transient absorption data assuming a series of two successive exponential steps, $A \rightarrow B \rightarrow$ (Bottom). $A$ can be assigned to $\mathbf{P e} \cdot{ }^{+}$in the $\mathrm{D}_{1}$ state and $B$ to the vibrationally hot $\mathrm{D}_{0}$ state.

Incidentally, the $\mathrm{D}_{1}$ lifetime of the radical anion of perylene, $\mathbf{P e}^{\cdot^{-}}$, also produced electrochemically in acetonitrile, amounts to

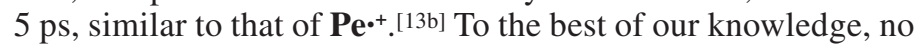
other radical anion of PAHs has been investigated so far with ultrafast spectroscopy, and, thus, no real conclusion can be drawn. It should nevertheless be noted that this 5 ps time constant is much shorter than those of 530 and 150 ps reported for the radical anions of the perylene monoimide and diimide. ${ }^{[22]}$ The origin of this difference is still unclear.

The excited-state dynamics of both $\mathbf{P e}^{\mathbf{}^{+}}$and $\mathbf{P e}^{\mathbf{-}^{-}}$in liquids was also investigated using pump-pump-probe spectroscopy. ${ }^{[13]}$ Interestingly, the excited lifetime of these two ions was found to depend on their 'age', i.e. on the time delay, $\Delta \mathrm{t}_{12}$, between the first actinic pump pulse that triggers their formation by bimolecular photoinduced ET and the second pump pulse that excites the ensuing radical ion (Fig. 1). For example, the excited-state

lifetime of $\mathbf{P e}^{-{ }^{-}}$generated upon ET with dimethylaniline increases from $1.2 \mathrm{ps}$ at $\Delta \mathrm{t}_{12}=250 \mathrm{ps}$ to $3 \mathrm{ps}$ at the highest achievable $\Delta t_{12}$ value of 2 ns.[13b] We could show that this effect originates from a charge recombination process between the excited ion and the corresponding counter-ion, leading to the population of the neutral perylene in the $S_{1}$ state. This additional decay pathway is only operative when the ions are paired and therefore the increasing excited-state lifetime with $\Delta \mathrm{t}_{12}$ reflects the increasing distance between the geminate ions. This sensitivity to the interionic distance could be used in the future as a kind of molecular ruler to monitor the temporal evolution of the distance distribution of the photoinduced ET product.

\section{The Radical Cations of Heterocycles}

We finish this overview by discussing the excited-state dynamics of the radical cation of three heterocyclic compounds, namely the tetrathiafulvalene $\left(\mathbf{T T F}{ }^{+}\right)$, thiantrene $\left(\mathbf{T H}^{*}\right)$ and $\mathrm{N}$-methylphenothiazine (MPTZ ${ }^{+}$) cations (Fig. 7). ${ }^{[12]}$ The reason to select these radical cations was that stationary emission had been reported for all of them, suggesting an excited-state lifetime that is relatively longer than for the above-discussed ions.<smiles>C1=CSC(=C2SCCS2)S1</smiles>

TTF

electrochem. $0.8 \mathrm{ps}$

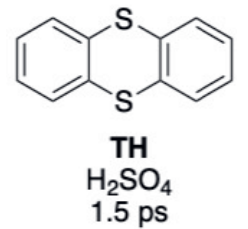

Fig. 7. Structure of the heterocyclic compounds, whose radical cations (formed in sulphuric acid or electrochemically) were investigated, together with the excited-state lifetimes in liquids at room temperature determined by ultrafast spectroscopy.

Schweig and coworkers reported distinct stationary fluorescence from $\mathbf{T T F}{ }^{*}{ }^{+}$and two derivatives in solid matrices at $77 \mathrm{~K} .{ }^{[23]}$ However, in agreement with the absence of fluorescence at room temperature, our transient absorption measurements of TTF.+ produced electrochemically in acetonitrile revealed dynamics with $\mathrm{D}_{1} \rightarrow \mathrm{D}_{0}$ internal conversion occurring in less than 1 ps (Fig. 8), very similar to those observed with the above-mentioned cations. The strong temperature dependence of the fluorescence quantum yield of $\mathbf{T T F}^{*}{ }^{+}$bears some resemblance to that of Wurster's Blue (TMPD ${ }^{+}$) discussed above and is consistent with the presence of a $D_{1} / D_{0}$ conical intersection separated from the Franck-Condon region of the $\mathrm{D}_{1}$ state by a small barrier. This calls for a more detailed study of the temperature dependence of the excited-state dynamics of this cation. However, this requires using another method than electrochemistry for generating $\mathbf{T T F}^{\cdot+}$.

By contrast, $\mathbf{T H}^{+}{ }^{+}$prepared in a $45: 5: 1$ dichloromethane/trifluoroacetic anhydride/trifluoroacetic acid mixture was reported to fluoresce at room temperature around $580 \mathrm{~nm}$ with a $4.7 \mathrm{~ns}$ lifetime. ${ }^{[24]}$ The ET quenching of its fluorescence by various donors was found to have a driving-force dependence that significantly departs from that usually found for the fluorescence quenching of closed-shell neutral molecules. ${ }^{[25]}$

Surprisingly, no emission could be detected with $\mathbf{T H}{ }^{+}{ }^{+}$prepared in sulfuric acid and transient absorption measurement indicate a $\mathrm{D}_{1}$ lifetime of $\mathbf{T H}{ }^{+}$of $1.5 \mathrm{ps}^{\left[{ }^{[12]}\right.}$ As the $\mathrm{D}_{1} \leftarrow \mathrm{D}_{0}$ absorption band of $\mathbf{T H}^{+}{ }^{+}$peaks at $1070 \mathrm{~nm}$, emission at $580 \mathrm{~nm}$ should correspond to a fluorescence from the $\mathrm{D}_{3}$ state, which is not expected to have a nanosecond lifetime, as reported. This emission is most probably due to a secondary product present in the solution and not to $\mathbf{T H}{ }^{+}$. Similar emissive side products were reported upon 


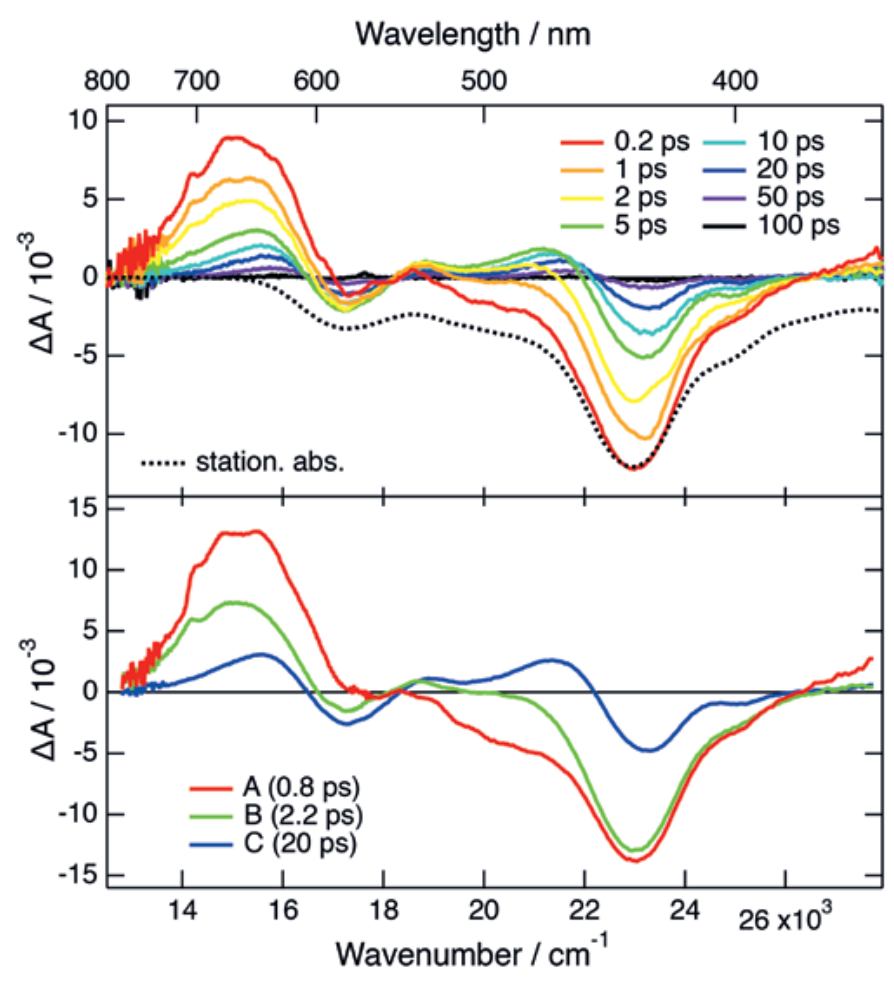

Fig. 8. Transient absorption spectra recorded at various time delays after $500 \mathrm{~nm}$ excitation of TTF.+ produced electrochemically in acetonitrile and negative stationary absorption spectrum (Top). Evolution-associated difference absorption spectra (EADS) and corresponding time constants obtained from a global analysis of the transient absorption data assuming a series of three successive exponential steps, $A \rightarrow B \rightarrow C \rightarrow$ (Bottom). $A$ can be assigned to TTF.+ in the $D_{1}$ state, and $B$ and $C$ to the vibrationally hot $D_{0}$ state. Some contribution of the $D_{1}$ state to EADS $B$ arising from non-exponential dynamics cannot be excluded.

electrochemical generation of radical ions. ${ }^{[26]}$

Finally, MPTZ $*^{+}$, prepared by oxidation with iodine, was also reported to emit in the visible, with a maximum at $600 \mathrm{~nm} .{ }^{[27]}$ As the lowest-energy absorption band of this cation is around $830 \mathrm{~nm}$, this emission should also originate from an upper excited state. This is somewhat consistent with the reported fluorescence quantum yield of only $3 \cdot 10^{-4}$, pointing to an efficient non-radiative decay of the excited state. Transient absorption measurements performed upon $\mathrm{D}_{\mathrm{n}>1} \leftarrow \mathrm{D}_{0}$ excitation of MPTZ.+ in concentrated sulfuric acid indeed reveal that the ground state is rapidly repopulated with a 26 ps time constant. ${ }^{[12]}$ Whereas the transient spectra show clearly the bleach of the intense $\mathrm{D}_{\mathrm{n}>1} \leftarrow \mathrm{D}_{0}$ absorption band at $510 \mathrm{~nm}$, they do not exhibit any spectral feature that could be associated with the corresponding $\mathrm{D}_{\mathrm{n}>1} \rightarrow \mathrm{D}_{0}$ stimulated emission. Based on this, one can conclude that internal conversion from the photo-populated $\mathrm{D}_{\mathrm{n}>1}$ state to the $\mathrm{D}_{1}$ state occurs within the $200 \mathrm{fs}$ instrument response function of the setup, as expected, and that the 26 ps time constant corresponds to the $\mathrm{D}_{1} \rightarrow \mathrm{D}_{0}$ internal conversion. Therefore, the weak $600 \mathrm{~nm}$ emission most probably originates from another species than MPTZ •+.

\section{Relevance of these Results}

All the open-shell ions investigated so far in our group are characterised by a very short excited-state lifetime ranging from a few hundreds of fs to less than 30 ps. These results are consistent with other studies of radical cations ${ }^{[28]}$ and anions ${ }^{[22,29]}$ performed with sufficient time resolution. The radical anions of naphthalene diimides and perylene mono- and diimides as well as the radical cation of peri-xanthenoxanthene are exceptional with an excited-state lifetime between 100 and 500 ps in solution. ${ }^{[22,28 e, 30]}$ To the best of our knowledge, the other longer lifetimes in liquids have been determined either indirectly, or from measurements with insufficient time resolution, or from time-resolved fluorescence, which, as discussed above, could originate from other species. These ultrafast excited-state dynamics of radical ions most probably stem from their flexibility, that increases first by going from the neutral to the ionic form, and second upon optical excitation. This, together with the small $\mathrm{D}_{1}-\mathrm{D}_{0}$ gap at the $\mathrm{D}_{0}$ equilibrium geometry, should favour the existence of a relatively large number of $\mathrm{D}_{1} / \mathrm{D}_{0} \mathrm{CIs}$, among which at least one can be efficiently accessed from the Franck-Condon region of the $\mathrm{D}_{1}$ state at room temperature.

These short lifetimes are clearly detrimental for the applications of excited ions as super-redox agents. For example, efficient quenching of an excited ion with a 10 ps lifetime requires the charge-transfer process to have an intrinsic rate constant above $10^{11} \mathrm{~s}^{-1}$ and a quencher concentration large enough, typically $>0.5 \mathrm{M}$, to ensure diffusive encounter while the ion is excited. Given the 200-300 fs excited state lifetime of the $p$-phenylenediamine cations, ${ }^{[8 c]}$ one can wonder whether the oxidation of benzyl alcohol to benzaldehyde reported by Moutet and Reverdy occurs via an electron transfer with $\mathrm{N}, \mathrm{N}, \mathrm{N}^{\prime}, \mathrm{N}^{\prime}-$ tetraphenyl- $p$-phenylenediamine in the $\mathrm{D}_{1}$ state as proposed, ${ }^{[2]}$ or rather involves a reaction with a longer-lived side product.

These ultrashort lifetimes make the detection of excited ions as products of highly exergonic ET reactions problematic. For example, TMPD is a very strong donor and consequently its radical cation can be expected to be populated in the $\mathrm{D}_{1}$ state upon photoinduced ET with a strong electron acceptor like e.g. tetracyanoanthracene (TCA, Fig. 9). If the bimolecular ET reaction after excitation of TCA yields TMPD ${ }^{+*}$, its detection in a transient absorption experiment is extremely challenging, to say the least, given the $\mathrm{D}_{1}$ state lifetime of less than $300 \mathrm{fs}$. Detection of TMPD+** using time-resolved fluorescence might be a better approach but requires extreme caution to ensure that the emission indeed arises from this species. Fig. 9 indicates that bimolecular ET could also yield TCA $^{-}$in the $\mathrm{D}_{1}$ state. However, we recently found that the excited-state of $\mathbf{T C A}^{-{ }^{-}}$is short lived as well and that its radiative decay should take place in the near IR.[31]

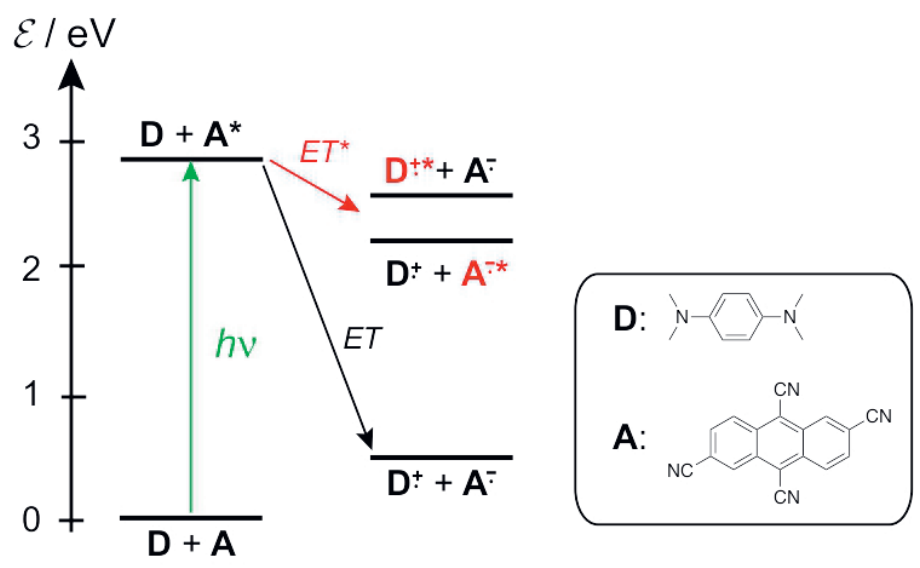

Fig. 9. Energy-level scheme pertaining to the photoinduced electron transfer between tetracyanoanthracene and TMPD. Electron transfer to the ion pair in the electronic ground state $(E T)$ is highly exergonic and should be in the Marcus inverted region. Electron transfer to the excited ions $\left(E T^{*}\right)$ is less exergonic and should be faster.

Lacking unambiguous detection of the excited ions, our observation of an asymmetric distribution of the energy dissipated in the donor and the acceptor upon highly exergonic ET remains the best evidence of the involvement of excited ions. ${ }^{[32]}$ 


\section{Acknowledgments}

We thank Prof. Paul Low (University of Western Australia) for providing us with a sample of DMTPD·+ salt. We express our gratitude the Swiss National Science Foundation (current project 200020-184607) as well as the University of Geneva for their continuous financial support.

Received: June 28, 2021

[1] a) E. Haselbach, T. Bally, Pure \& Appl. Chem. 1984, 56, 1203, http://dx.doi.org/10.1351/pac198456091203; b) S. S. Shukla, J. F. Rusling, J. Phys. Chem. 1985, 89, 2253

[2] J.-C. Moutet, G. Reverdy, Chem. Commun. 1982, 654 , https://doi.org/10.1039/C39820000654.

[3] a) I. Ghosh, T. Ghosh, J. I. Bardagi, B. König, Science 2014, 346, 725, https://doi.org/10.1126/science.1258232; b) M Fujitsuka, T. Majima, J. Photochem. Photobiol. C 2018, 35, 25, https://doi.org/10.1016/j.jphotochemrev.2017.12.003; c) J. A. Christensen, B. T. Phelan, S. Chaudhuri, A. Acharya, V. S. Batista, M. R. Wasielewski, J. Am. Chem. Soc. 2018, 140, 5290, https://doi.org/10.1021/jacs.8b01778; d) J. P. Barham, B. König, Angew. Chem. Int. Ed. 2020, 59, 11732, https://doi.org/10.1002/anie.201913767; e) H. Kim, H. Kim, T. H. Lambert, S. Lin, J. Am. Chem. Soc. 2020, 142, 2087, https://doi.org/10.1021/ jacs.9b10678; f) F. Glaser, C. Kerzig, O. S. Wenger, Angew. Chem. Int. Ed. 2020, 59, 10266, https://doi.org/10.1002/anie.201915762.

[4] a) D. Rehm, A. Weller, Isr. J. Chem. 1970, 8, 259, https://doi.org/10.1002/ijch.197000029; b) A. I. Burshtein, A. I. Ivanov, Phys. Chem. Chem. Phys. 2007, 9, 396, https://doi.org/10.1039/B610146A; c) A. Rosspeintner, G. Angulo, E. Vauthey, J. Am. Chem. Soc. 2014, 136, 2026, https://doi.org/10.1021/ja4118279; d) R. A. Holroyd, J. R. Miller, J. Phys. Chem. B 2019, 123, 9206, https://doi.org/10.1021/acs.jpcb.9b07845.

[5] a) T. Shida, E. Haselbach, T. Bally, Acc. Chem. Res. 1984, 17, 186, https://doi.org/10.1021/ar00101a005; b) T. Shida, 'Electronic Absorption Spectra of Radical Ions', vol. 34, Elsevier, Amsterdam, 1988.

[6] T. Bally, Chimia 1994, 48, 378.

[7] a) J.-C. Gumy, E. Vauthey, J. Phys. Chem. A 1997, 101, 8575, https://doi.org/10.1021/jp972066v; b) P. Brodard, A. Sarbach, J.C. Gumy, T. Bally, E. Vauthey, J. Phys. Chem. A 2001, 105, 6594, https://doi.org/10.1021/jp0108081.

[8] a) L. Michaelis, M. P. Schubert, S. Granick, J. Am. Chem. Soc. 1939, 61, 1981, https://doi.org/10.1021/ja01877a013; b) P. J. Low, M. A. J. Paterson, H. Puschmann, A. E. Goeta, J. A. K. Howard, C. Lambert, J. C. Cherryman, D. R. Tackley, S. Leeming, B. Brown, Chem. Eur. J. 2004, 10, 83, https://doi.org/10.1002/chem.200305200; c) J. Grilj, P. Buchgraber, E. Vauthey, J. Phys. Chem. A 2012, 116, 7516, https://doi.org/10.1021/jp3045548.

[9] W. I. Aalbersberg, G. J. Hoijtink, E. L. Mackor, W. P. Weijland, J. Chem. Soc. 1959, 3049, https://doi.org/10.1039/JR9590003049.

[10] M. Krejčik, M. Daněk, F. Hartl, J. Electroanal. Chem. Interf. Electrochem. 1991, 317, 179, https://doi.org/10.1016/0022-0728(91)85012-E

[11] a) H. Larsen, S. U. Pedersen, J. A. Pedersen, H. Lund, J. Electroanal. Chem. 1992, 331, 971, https://doi.org/10.1016/0022-0728(92)85018-X; b) B Mladenova, R. Kattnig Daniel, G. Grampp, Z. Phys. Chem. 2006, 220, 543, https://doi.org/10.1524/zpch.2006.220.4.543.

[12] J. Grilj, PhD thesis, University of Geneva, $\mathrm{nr}$ 4326, 2011

[13] a) S. Pagès, B. Lang, E. Vauthey, J. Phys. Chem. A 2006, 110, 7547, https://doi.org/10.1021/jp0615252; b) J. S. Beckwith, B. Lang, J. Grilj, E. Vauthey, J. Phys. Chem. Lett. 2019, 10, 3688, https://doi.org/10.1021/acs.jpclett.9b01431.

[14] J. S. Beckwith, A. Rosspeintner, G. Licari, M. Lunzer, B. Holzer, J. Fröhlich, E. Vauthey, J. Phys. Chem. Lett. 2017, 8, 5878, https://doi.org/10.1021/acs.jpclett.7b02754.

[15] J. Grilj, E. N. Laricheva, M. Olivucci, E. Vauthey, Angew. Chem. Int. Ed. 2011, 50, 4496, https://doi.org/10.1002/anie.201100015.

[16] a) V. Sundström, T. Gillbro, H. Bergström, Chem. Phys. 1982, 73, 439, https://doi.org/10.1016/0301-0104(82)85183-5; b) Y. Nagasawa, Y. Ando, D Kataoka, H. Matsuda, H. Miyasaka, T. Okada, J. Phys. Chem. A 2002, 106, 2024, https://doi.org/10.1021/jp012135c; c) P. Fita, A. Punzi, E. Vauthey, $J$. Phys. Chem. C 2009, 113, 20705, https://doi.org/10.1021/jp906676x.
[17] a) T. A. Miller, Annu. Rev. Phys. Chem. 1982, 33, 257; b) J. P. Maier, Int. J. of Mass Spectrom. Ion Proces. 1991, 104, 1.

[18] a) P. J. Sarre, J. Mol. Spectrosc. 2006, 238, 1 , https://doi.org/10.1016/j.jms.2006.03.009; b) D. K. Bohme, Chem. Rev. 1992, 92, 1487, https://doi.org/10.1021/cr00015a002; c) T. P. Snow, V. L. Page, Y. Keheyan, V. M. Bierbaum, Nature 1998, 391, 259, https://doi.org/10.1038/34602.

[19] L. Zhao, R. Lian, I. A. Shkrob, R. A. Crowell, S. Pommeret, E. L. Chronister, A. D. Liu, A. D. Trifunac, J. Phys. Chem. A 2004, 108, 25, https://doi.org/10.1021/jp021832h

[20] a) K. F. Hall, M. Boggio-Pasqua, M. J. Bearpark, M. A. Robb, J. Phys. Chem. A 2006, 110, 13591, https://doi.org/10.1021/jp064711g; b) A. M Tokmachev, M. Boggio-Pasqua, M. J. Bearpark, M. A. Robb, J. Phys. Chem. A 2008, 112, 10881, https://doi.org/10.1021/jp8044109.

[21] A. M. Tokmachev, M. Boggio-Pasqua, D. Mendive-Tapia, M J. Bearpark, M. A. Robb, J. Chem. Phys. 2010, 132, 044306, https://doi.org/10.1063/1.3278545.

[22] D. Gosztola, M. P. Niemczik, W. Svec, A. S. Lukas, M. R. Wasielewski, J. Phys. Chem. A 2000, 104, 6545, https://doi.org/10.1021/jp000706f.

[23] K. Zimmer, B. Gödicke, M. Hoppmeier, H. Meyer, A. Schweig, Chem. Phys. 1999, 248, 263, https://doi.org/10.1016/S0301-0104(99)00246-3.

[24] J. Eriksen, H. Lund, A. Nyvad, Acta Chem. Scand. 1983, B37, 459, https://doi.org/10.3891/acta.chem.scand.37b-0459.

[25] J. Eriksen, K. A. Joergensen, J. Linderberg, H. Lund, J. Am. Chem. Soc. 1984, 106, 5083, https://doi.org/10.1021/ja00330a008.

[26] D. T. Breslin, M. A. Fox, J. Phys. Chem. 1994, 98, 408, https://doi.org/10.1021/j100053a011.

[27] H. Shine, D.-C. Zhao, J. Org. Chem. 1990, 55, 4086 , https://doi.org/10.1021/jo00300a026.

[28] a) Y. Huang, J. B. Hopkins, J. Phys. Chem. A 1996, 100, 9585, https://doi.org/10.1021/jp9520198; b) T. Haeupl, R. Lomoth, L. Hammarström, J. Phys. Chem. A 2003, 107, 435, https://doi.org/10.1021/jp0216023; c) A. N. Okhrimenko, A V. Gusev, M. A. J. Rodgers, J. Phys. Chem. A 2005, 109, 7653, https://doi.org/10.1021/jp053136y; d) S. Amarie, U. Förster, N. Gildenhoff, A. Dreuw, J. Wachtveitl, Chem. Phys. 2010, 373, 8, https://doi.org/10.1016/j.chemphys.2010.01.017; e) J. A. Christensen, J. Zhang, J. Zhou, J. N. Nelson, M. R. Wasielewski, J. Phys. Chem. C 2018 122, 23364, https://doi.org/10.1021/jacs.8b01778.

[29] a) M. J. Hope, M. P. Higlett, D. L. Andrews, S. R. Meech, I. D Hands, J. L. Dunn, C. A. Bates, Chem. Phys. Lett. 2009, 474, 112, https://doi.org/10.1016/j.cplett.2009.04.066; b) M. Fujitsuka, S. S. Kim, C. Lu, S. Tojo, T. Majima, J. Phys. Chem. B 2015, 119, 7275, https://doi.org/10.1021/jp510850z.

[30] a) M. Fujitsuka, T. Ohsaka, T. Majima, Phys. Chem. Chem. Phys. 2015, 17, 31030, https://doi.org/10.1039/C5CP05254H; b) C. Lu, M Fujitsuka, A. Sugimoto, T. Majima, J. Phys. Chem. C 2017, 121, 4558 , https://doi.org/10.1021/acs.jpcc.7b00970.

[31] J. S. Beckwith, PhD thesis, University of Geneva, nr 5401, 2019.

[32]M. Koch, A. Rosspeintner, K. Adamczyk, B. Lang, J. Dreyer, E. T. J. Nibbering, E. Vauthey, J. Am. Chem. Soc. 2013, 135, 9843, https://doi.org/10.1021/ja403481v.

\section{License and Terms}

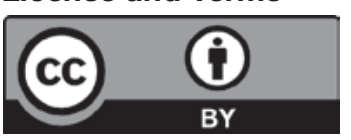

This is an Open Access article under the terms of the Creative Commons Attribution License CC BY 4.0. The material may not be used for commercial purposes.

The license is subject to the CHIMIA terms and conditions: (http:// chimia.ch/component/sppagebuilder/?view=page \&id $=12$ ).

The definitive version of this article is the electronic one that can be found at https://doi.org/10.2533/chimia.2021.856 